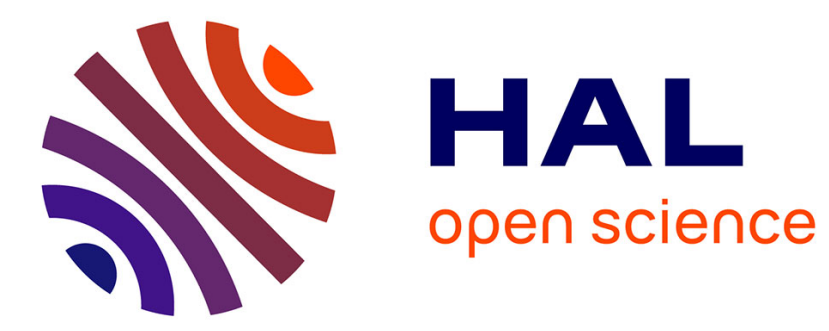

\title{
La Formation de la Mamora. Le point sur la question du Moulouyen et du Salétien du Maroc Nord-Occidental.
}

Pierre-Jean Texier, David Lefevre, Jean-Paul Raynal

\section{To cite this version:}

Pierre-Jean Texier, David Lefevre, Jean-Paul Raynal. La Formation de la Mamora. Le point sur la question du Moulouyen et du Salétien du Maroc Nord-Occidental.. Quaternaire, 1993, 3 (2), pp.63-73. halshs-00004650

\author{
HAL Id: halshs-00004650 \\ https://shs.hal.science/halshs-00004650
}

Submitted on 17 Sep 2005

HAL is a multi-disciplinary open access archive for the deposit and dissemination of scientific research documents, whether they are published or not. The documents may come from teaching and research institutions in France or abroad, or from public or private research centers.
L'archive ouverte pluridisciplinaire HAL, est destinée au dépôt et à la diffusion de documents scientifiques de niveau recherche, publiés ou non, émanant des établissements d'enseignement et de recherche français ou étrangers, des laboratoires publics ou privés. 


\title{
LA FORMATION DE LA MAMORA Le point sur la question du Moulouyen et du Salétien du Maroc Nord-Occidental
}

\author{
Jean-Pierre TEXIER*, David LEFEVRE** et Jean-Paul RAYNAL*
}

RESUME

La révision des coupes-types et l'étude de nouvelles stratigraphies de la «Formation de la Mamora» (Maroc nord occidental) nous conduisent à rejeter l'existence du néo-stratotype du Moulouyen el du stratotype de Salétien, considérés comme les deux premiers étages continentaux du Quaternaire marocain. En effet, les dépôts ayant servi à leur définition correspondent en grande partie à des altérites non remaniées, pédogénisées (Moulouyen) et localement ferruginisées (Salétien) de formations pliocènes. En revanche, la partic supérieure de cette formation, constituée de dépôts colluviaux et ́́oliens, a enregistré toute une série d’évènements morpho-pédo-sédimentaires pléstocènes. Deux unités principales peuvent y être dístinguées: des sables beiges de couverture, d'origine éolienne et datés de 28 à $10 \mathrm{Ka}$; un complexe de sables et argiles rouges panachés dont l'âge peut aller du début du Pléistocène moyen à la partie supérieure oú dernier cycle climatique.

Mots-elés: Maroc nord-oxcidental, Pliocène, Pléistucène, stratigraphie, stratolypes, Moulouyen, Salétien, enregistrements, paléoenvitonnements.

\section{ABSTRACT}

THE MARMORA FORMATION. THOUGHTS ON THE MOULOUYAN AND SALETIAN STAGES OF NORTH-WESTERN MAROC$\mathrm{CO}$

The revision of classic stratigraphic scctions of the Mamora region (Nurthwestern Morocco) and the study of new exposures located in the same area show that the first two continental stages of the Moroccan Quatcrnary arc incorrectly identified and that the terms of «Moulouyan and «Saletian» must be rejected. Deposits used for their definition are in fact in situ Pliocene sediments which are wcathcred (= Moulouyan») and locally ferruginised (= $=$ Saletian»)

In contrast, the upper part of the «Mamora Formation», consisting of colluvial and eolian deposits, is of Pleistocene age and has reconded several morpho-pedological events. Two main stratigraphic units can be distinguished: 1) a sheet of light brown eolian sands ( (sables beiges» of the terminology) dated between 28 and $10 \mathrm{Ky}$, and 2) a sedimentary complex of streaked red sands and clays. This last unit can be approximately dated between the beginning of the Middle Pleistocene and the upper part of the last climatic cycle.

Key-words: North-Western Marocco, Pliocene, Pleistocene, stratigraphy, stratotypcs, Moulouyan, Saletian, recorưs, environments.

\section{I - INTRODUCTION}

Les plateaux formant la terminaison septentrionale de la Meseta marocaine sont recouverts par une formation sablo-argileuse rouge connue sous le nom de "Formation de la Mamora".

Interprétée comme un épandage fluviatile rubéfié ravinant les formations moghrébiennes sous-jacentes, la «Formation de la Mamora» a été rapportée au
Moulouyen (Choubert, 1957-65a; Gigout, 1958, 1960; Gigout et Raynal, 1957, 1959), premier «pluvial-étagc» de la stratigraphie du Quaternaire continental marocain (Choubert et al., 1956). En divers points de la région, elle a fourni des industries préhistoriques considérées comme très anciennes (Biberson, 1961). Le site de Douar Doum, près de Rabat, aujourd'hui inaccessible, a été proposé pour définir le néo-stratotype du Moulouyen pour la région atlantique.(Biberson, 1971). Pour

- Institut du Quatemaire, URA 133 du C.N.R.S., Université de Burdeaux I, Avenue des Facultés, F-3340S TALENCE Cedex.

*" Laboratoire de Géomorphologie et d'Etude du Quaternairc, Université des Sciences el Technologies de Lille, F-59655 VILLENEUVE D'ASCO Cedex.

*** Mission paléontologique et préhistorique française au Maroce 
les auteurs, le Moulouyen est un étage correspondant soit au Villafranchien supérieur (Choubert, 1957-65a Gigout, 1958, 1960; Gigout et Raynal, 1957, 1959; André et Beaudet, 1967; Beaudet et al., 1967; Beaudet, 1971) soit au Villafranchien moyen (Biberson, 1961, 1971; Choubert, 1965).

Le Coz (1964) interprète les différents niveaux observés en Mamora comme des sous-étaues du Villafranchien: à la base, formation argilo-sableuse à galets du Villafranchien inférieur; puis sables rouges du Villafranchicn moycn et, au sommet, sables argileux tachetés du Moulouyen; les sables supcrficiels résulteraient de remaniements post-villafranchiens.

En revanche, Faraj (1963) puis Lepoutre (1966-68) ne distinguent que deux ensembles: un ensemble inférieur sablo-argileux rouge correspondant à des sols développés sur les grès dunaires, et un ensemble superieur sableux beige, d'épaisseur variable, d'origine éolienne pour Faraj, fluviatile pour Lepoutre.

Saaïdi (1974) y reconnaît également deux ensembles: à la base, la Formation Rouge sensu stricto, qu'il interprète comme un dépôt marin datant du Miocène et du Plio-Quaternaire, rubéfié sous un climat chaud et humide au cours du Villafranchien moyen et supérieur; au sommet, une couche sableuse beige, d'âge fini ou postvillafranchien, provenant d'anciennes plages émergées et remaniées par le vent.

Localement, sur le plateau de Salé, une nappe de galets fortement ferruginisée ravinant la *Formation de la Mamora» (le Moulouyen) a été retenue comme le stratotype du Salétien (Choubert et al., 1956). Les sables beiges superficiels à pisolithes ferrugineux ont été interprétés commé ses équivalents latéraux (André et Beau. det, 1967; Beaudet, 1969). Le Salétien représenterait le "premier pluvial quaternaire post-villafranchien" (Choubert et al., 1956; Choubert, 1957-65a; Beaudet, 1969, 1971). Lcs formations salétiennes, qui ont livré des objets préhistoriques attribués au «Chelléen» (Chou- bert et Roche, 1956) ou au «Pré-Acheuléen évolué» (Biberson, 1961) sont en revanche rapportées au Villafranchien supérieur par Biberson $(1961,1971)$ et Choubert (1965).

La signification et la place des étages Moulouyen et Salétien dans la stratigraphie du Quaternaire continental marocain ont fait l'objet de controverses. Celles-ci furent compliquées du fait de l'utilisation par les auteurs du terme Villafranchien, dans le sens chronostratigraphique d'étage intercalé entre le Pliocène et le Pléistocène/Quaternaire, mais avec des acceptions très variées pour ce qui concerne les limites, et non dans le sens d'associations faunistiques (Lhénaff Ed., 1983).

Les travaux réalisés dans le cadre de la Mission préhistorique et paléontologique française au Maroc nous ont amené à réexaminer ces formations régionales. Il est rapidement apparu que les diverses interprétations proposées ne s'accordaient ni avec une analyse pédo-stratigraphique fine des coupes-types, ni avec les caractéristiques des assemblages archéologiques identifiés a différents niveaux dans ces dépôts.

Une révision d'ensemble s'imposait donc pour déterminer la part respective des processus sédimentaires et pédologiques intervenus lors de la mise en place de cette couverture et tenter de situer chronologiquement les différents événements identifiés.

Les premiers résultats ont montré que ces formations étaient polygeniques et complexes, leur histoire se déroulant tout au long du Quaternaire (Texier et al., 1982; Raynal et al., 1982; El Hajraoui et al., 1984; Texier et al., 1984; Raynal et al., 1985).

L'avancement des travaux permet aujourd'huide faire un point d'ensemble, de proposer une interprétation plus approfondie des différents processus morpho-pédosédimentaires et d'en tirer les conséquences du point de vue de la stratigraphie du Quaternaire continental marocain.


Fig. 1: La Mamora. Localisation et cadre gémorphologique. 1: versants de vallées, 2: morphologie en cordons dunaires, 3: formations
sableuses et sablo-argileuses de couverture (Formation de la Mamora), 4t sites étudiés ou mentionnés Rouge I et II, 3: Sale-carrefour, 4: Salé-Aîn es SaÁ, 5: Salé-Bayleville, 6 : Daya el Hamra, 7: Tardiguet or Rhala. 


\section{2 - CADRE GEOGRAPHIQUE ET GEOLOGIQUE}

D'une superficie de $2300 \mathrm{~km}^{2}$, la Mamora est un vaste plateau situe à la limite septentrionale de la basse Meseta (fig. 1). Dans sa partie occidentale, elle est constituée d'une succession de rides et de dépressions allongées selon un axe SW-NE, parallèlement au littoral. Ces ondulations s'atténuent vers l'est et passent à un plateau dont la surface relativement plane s'abaisse progressivement vers le nord en direction de la plaine du Gharb. Lc rćseau hydrographique, encaissé dans d'étroites vallécs, épousc cc modclé ct s'organise en éléments également orientés SW-NE.

La région qui appartient au domaine du climat océanique, à la limite des étages sub-humide, le long du littoral, et semi-aride, est marquée par un gradient d'aridité croissant vers l'est. Elle est recouverte par une chênaie à Quercus suber en peuplement pur, avec Pynıs mamorensir épars et Genista linifolia en sous-bois (Lepoutre, 1966).

Absente sur une étroite frange côtière, la «Formation de la Mamora" repose sur un substratum classiquement désigné sous le terme de «Moghrébien» (Choubert, 1957-65b). Celui-ci est composé principalement de calcarénites bioclastiques ou de conglomérats à matrice calcaire correspondant à des environnements littoraux et fluvio-deltaiques (Cirac, 1985). Localement, notamment sur le plateau de Salé, s'intercale vers le haut de la série, un banc d'argiles blanches a débit polyédrique, de a a 2,5 m d'épaisseur. La base de la série correspond à la «dalle moghrébienne" des auteurs, banc de grès grossiers, biodétritiques, parfois lumachelliques, epais de $0,5 \mathrm{~m}$ à $1 \mathrm{~m}$, de faciès marin littoral, qui appartient la biozone à Globorotalia crassaformis, d'âge Pliocène moyen (Wernli, 1978).

\section{3 - MATERIAUX ET METHODES}

L'étude a porté sur un certain nombre de coupes types répartics dans trois secteurs de la Mamora: le plateau de Rabat, le plateau de Salć ct lc platcau de Tiflet (fig. 1) Celles-ci comprennent non seulement les stratigraphics classiques (Salé-Aïn Saâ, Tardiguet-er-Rhala), mais aussi d'autres coupes susceptibles d'apporter un éclairage nouveau sur les problèmes posés par ces formations régionales.

Nous avons appliqué les règles formelles de la lithostratigraphie et de la chronostratigraphie (Hedberg, 1976) aux séquences sédimentaires quaternaires (Rat, 1980) Pour ce qui concerne les termes Pléistocène et Pliocène nous nous référons aux sens, limites et subdivisions définis par Bonifay (1975) et Texier et al. (1985).

Les méthodes utilisées sont principalement descriptives: morphologiques, litho-pedo-stratigraphiques et micromorphologiques. Les lames minces ont été décrites suivant la terminologie définie par Büllock et al. (1985). Des données provenant de travaux antérieurs (Texier et al., 1984; El Hajraoui, 1985; Aberkan, 1989; Lefèvre $e t a l ., 1991$ ) ont également été utilisées.

Par ailleurs, des datations numériques (Texier et al. 1988; Texier et Raynal, 1989; Rhodes, 1990; Smith et al., 1990; Raynal et al., 1992) et les éléments archéologiques inclus dans les formations étudiées (sites de Chaperon Rouge I et II, de Daya-el-Hamra, PK 13 de la route Rabat-Tiflet, Coupe Aloua près de Tiflet) ont fourni un certain nombre de repères chronologiques.

\section{4 - DONNEES STRATIGRAPHIQUES}

\section{1 - PLATEAU DE RABAT}

Deux coupes types, le Chaperon-Rouge I et II, ont été étudiées dans la partie sud-ouest du plateau de la Mamora, au sud de Rabat. Distantes, l'une de l'autre d'environ $2 \mathrm{~km}$, elles ont été réalisées sur des sites préhistoriques ayant fait l'objet de fouilles (Texier $e t a l$., 1982; Texier, 1985-86) et de datations numeriques (op. cit.).

Dans cette zonc, les formations superficielles com. prennent la succession stratigraphiquc suivantc, de haut en bas (fig. 2):

1 - Sables moyens à fins (Md entre 130 et 150 um) de couleur brun pâle, bien classés et comportant un pourcentage appréciable de grains ronds mats (20 à $30 \%)$. Epaisseur: 0,5 à $2-3 \mathrm{~m}$. Structure particulaire à massive. Vers la base, apparition de minces bandes plus argileuses (horizon I Bt), de couleur brun-rouge (2,5 YR 4/4). A Chaperon-Rouge I, ces dépôts repósent directement sur des calcarénites moghrébiennes par l'intermédiaire d'un horizon Béta.

A Chaperon-Rouge II, le contact avec les couches sous-jacentes est net, ondulé, parfois souligné par des éléments grossiers.

2 - Sables argileux de couleur jaune-rouge (5 YR 4/8 à 7/6). Epaisseur moyenne $0,15 \mathrm{~m}$. Structure prismatique très grossière. Présence de revêtements argileux peu exprimes de couleur brun-rouge (2,5 YR 4/4 à 5/8) et de sables de la couche 1 dans des biotubules. Limite inférieure nette.

Cette couche passe parfois latéralement à des structures ravinantes à remplissage complexe, de profondeur inférieure au mètre et de largeur supra-métrique. L'une d'elles dont la base est soulignée par des pisolithes ferrugineux remaniés comporte de haut en bas les niveaux suivants:

- Niveau sablo-argileux, épaisseur 0,40-0,50 m, rouge et massif au sommct, puis panaché, rouge, jaune et gris clair avec des traces de bioturbations ct, à la base, jaune et gris clair avec litage sub-horizontal visible localcment;

- Lentille argileuse d'épaisseur centimétrique et à débit finement polyédrique;

- Niveau massif, sablo-argileux. Epaisseur $0,30 \mathrm{~m}$. Couleur jaune au sommet devenant progressivement rouge vers la base.

3 - Sables argileux. Puissance observée 2,00 a $3,50 \mathrm{~m}$. On y distingue les horizons suivants:

- IIB21t: Bariolé, à couleurs dominantes rouge sombre (2,5 YR 4/8) et brun vif (7,5 YR 5/8). Epaisseur 0,15 $\mathrm{m}$. Structure polyédrique nette, fine. Limite inférieure distincte.

- IIB22tg: Structure prismatique moyenne. Epaisseur $0,25 \mathrm{~m}$. Unités structurales de couleur rouge sombre $(2,5$ YR 4/8) à l'intérieur et brun vif $(7,5$ YR 5/8) vers l'extérieur; sur les faces de ces unités, revêtements argileux rouge-jaune (5 YR 4/8), Existence de traînées verticales gris-clair (5 YR 7/1), larges de quelques centimètres, indépendantes de la structure et renfermant des revêtements argileux rouges (2,5 YR 4/6). Limite inférieure graduelle.

- IIB23tg: Structure prismatique grossière. Epaisseur $0,65 \mathrm{~m}$. Couleur rouge sombre (2,5 YR 4/8) dominante, brun vif (7,5 YR 5/8) sur quelques faces d'unités; revêtements argileux rouge-jaune (5 YR 4/8) aussi abondants qu'en B22t. Traînées verticales gris clair ( 5 YR 7/1) plus abondantes et plus épaisses, renfermant 

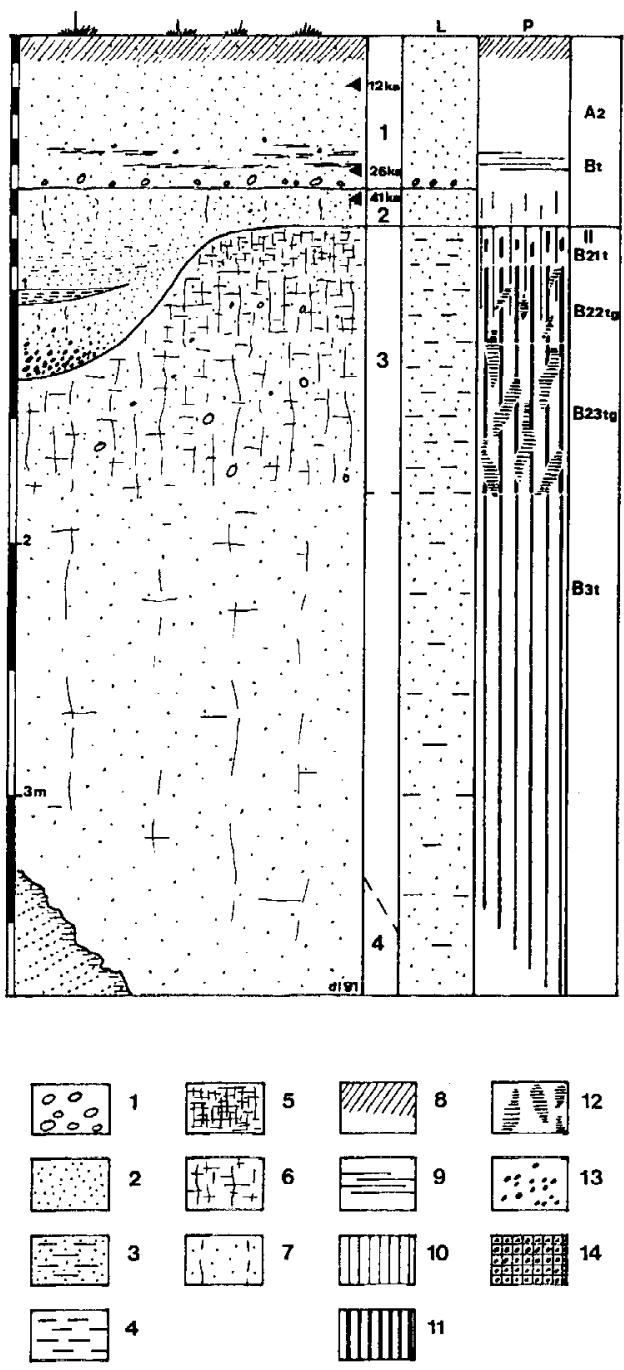

Fì. 2: Litho-pédostratigraphie de la coupe de Chaperon Rouge II. Légende des figurés utilisés dans les différentes coupess 1 eléments grossiers, 2: sables, 3: sables argileux, 4i argile, 5 horizon orano-minéral, stil prismatique, 7: str. massive, : argiliques faunes ou ocres, 11: vol, argiliques rouges, 12: vol. reduits, 13: pisolithes, 14: vol pétroferrique.

Fig.2: Litho-pedo-stratigraphical section of Chaperon Rouge II. 1: pebbles-gravels, 2: sands, 3; clayey sands, 4: clay, 5: polyedric smecture, 6: prismatic str., 7: massive str., 8: organo-mineral horizon, 9: band illuviation, 10: yellow argillic volumes, 11: red argillic vol. 12: redoxic vol, 13: pisolithes, 14: petro-feric vol.

également des revêtements argileux rouges (2,5 YR 4/ 6) et toujours indépcndantcs des taches brun vif et de la structure prismatique. Limite inféricurc distinctc.

II B 3t: Visible sur une épaisseur pluri-décimćtrique. Couleur rouge-jaune (5 YR 4/8). Structurc massive. Existence de nombreuses dendrites noires au sommet de l'horizon.
Le contact de l'horizon argilique avec les grès calcaires moghrébiens (4) est très irrégulier, de type karstique. On observe des poches de dissolution profondes de plusieurs mètres, séparées par des chicots carbonatés encore coherents. Le passage des sables argileux aux grès calcaires se fait par l'intermediaire d'un horizon à plages calcaires déjà pénétrées par la matrice argileuse rouge.

Localement, la structure prismatique de la couche 3 est déformée en éventail. Ce type de déformation est systématiquement associé à des soutirages des sables de surface susceptibles d'atteindre plusieurs mètres.

\section{2 - PLATEAU DE SALE}

Sur le plateau de Salé, les sables bruns à galets et pisolithes de la couche supérieure ont été rapportés an Salétien, les sables jaunes ou rouges sous-jacents au Moulouyen (Beaudet, 1969). Une coupe située près du carrefour de la route de Meknès, en haut de versant, permet d'observer de haut en bas la succession suivante (fig. 3):

1 - Sables friables, gris brun clair $(10$ YR $6 / 2)$ contenant de nombrcux pisolithes ferrugineux et des galets dispersés dans la masse. Epaisseur $0,60 \mathrm{~m}$. Contact inférieur brutal avec développement de petites glosses.

2 - Poches de largeur semi-métrique colmatées par des pisolithes ferrugineux inclus dans une matrice sablo-argileuse jaune brun (10 YR 6/6). Epaisseur variable jusqu'à $0,70 \mathrm{~m}$. Structure à support clastique. Localement, pisolithes cimentês par un ciment ferrugineux. Limite inférieure nette.

3 - Sables argileux, dans lesquels on distingue deux horizons:

- II B2tg, jaune brun (10 YR 6/6). Epaisseur 0,70 m. Présence de petites taches rouges (10 R 4/6) à contours diffus et de traînées verticales gris clair. Structure polyédrique bien exprimée. Revêtements argileux jaune sur les faces structurales. Nombreuses concrétions ferrugineuses. Limite inférieure progressive.

- II B3tg, panaché, rouge (10 R 4/6), gris clair (5 Y7/ 2) et jaune (10 YR 6/6). Epaisseur $0,35 \mathrm{~m}$. Structure polyédrique grossière mal exprimée. Surstructure prismatique. Revêtements gris clair sur les faces structurales. Présence de petites concrétions ferrugineuses. Limite inférieure diffuse.

4 - Argile à structure prismatique très grossière. Revêtements gris clair sur les éléments structuraux. Couleur brun jaune (10 YR 6/6) et gris clair ( 5 Y 7/2). Ce faciès correspond au banc d'argiles blanches qui s'intercale à la partie supérieure des calcarénites *moghrébiennes"; le contact est visible dans la carrière voisine dite Sale-cote $62 \mathrm{~m}$.

Dans la même région, à environ $1 \mathrm{~km}$ à l'est de Sale, se trouve la coupe classique d'Ain Es Saa, stratotype du Salétien (Choubert et al., 1956). Les dépôts ferruginisés et indurés qui y affleurent ont été distingués de la partie basale rapportée à la Fomation de la Mamora et interprétés comme représentant un cycle sédimentaire plus récent (Gigout, 1960; Beaudet, 1969). Cependant, dans la mesure où nous n'avons identifié aucun critère objectif justifiant cette séparation, nous la traitons dans le même cadre que la Fomation de la Mamora.

Elle est localisée à l'intérieur d'une légère dépression de largeur kilométrique qui s'ouvre vers le sud en direction de l'oued Bou Regreg. De haut en bas, on y observe (fig. 4);

1 - Galets dispersés dans une matrice ferruginisée 


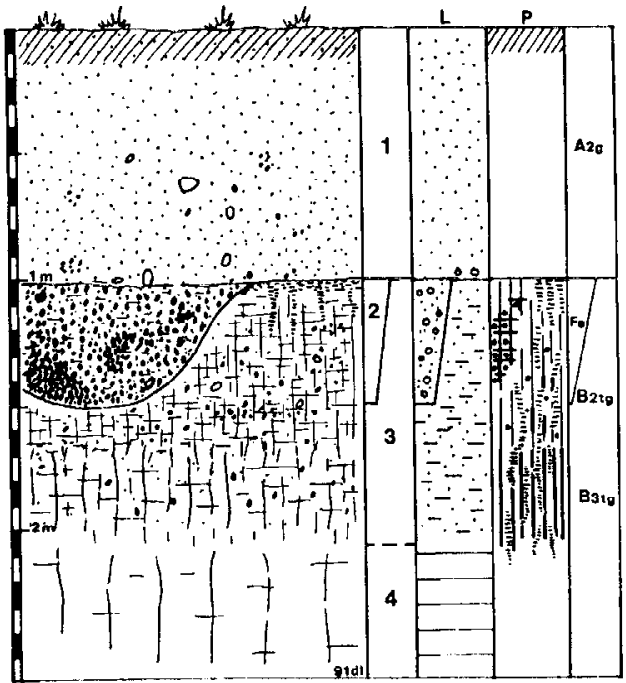

Fig 3: Litho-pédostratigraphle de la coupe de Salé-carrefour.

Fig. 3: Litho-pedostratigraphical section of Sale-carrefour.

fortement consolidée brun jaune foncé. Epaisseur 0,25 / $0,30 \mathrm{~m}$. Autour des galets, enrobement ferrugineux noir. Structure de type diamicton. Limite inférieure nette, régulière.

2 - Sables et galets siliceux organisés en lits à stratification entrecroisée du type cuillères emboîtées. Epaisseur visible sur $1,3 \mathrm{~m}$. La dalle ferrugineuse (ferricrète) qui affecte la couche 1, se développe également sur 40 à $60 \mathrm{~cm}$ dans la partie supérieure de 2. Dans les niveaux les plus fins, la ferruginisation souligne le litage. Localement, des «racines» à forte imprégnation ferrugineuse peuvent s'enfoncer jusqu'à $1,50 \mathrm{~m}$ sous la surface actuelle. La transition avec la partie inférieure de la couche est progressive. Celle-ci présente sur un fond de couleur rouge, des traînées gris clair parallèles au litage et recoupées par des traînées verticales jaunes.

Quelques kilometres plus à l'est, près de la ferme Bayleville, une coupe également située en haut de versant, montre la succession suivante (fig. 5):

1 - Sables gris brun clair, friables. Epaisseur 0,40 m.
Contact inférieur net, glossique.

2 - Sables argileux panachés rouges et jaune brun. Epaisseur $0,30 \mathrm{~m}$. Structure massive. Limite inférieure franche, marquée par une surface durcie affectée par des petites fentes.

3 - Sables argileux, massifs, jaune brun. Epaisseur $0,70 / 0,80 \mathrm{~m}$.

4 - Niveau de galets localement déformé et colmatant latéralement des petites dépressions de dimensions décimétriques a pluridécimétriques. Contact avec la couche inférieure de type érosif.

5 - Calcarénites altérées et pédogénisées, massives, cohérentes, comportant des diaclases sub-verticales colmatées par des dépôts ferro-manganiques. Couleur générale rouge et, vers le haut, larges traînées verticales jaune brun.

\section{3 - PLATEAU DE TIFLET}

Le site de Daya el Hamra, situé au NW de Tiflet, a livré une abondante industrie palécolithique attribuce à l'Acheuléen (El Hajraoui, 1985).

C'est dans la partic oricntale du gisement que les dépôts montrent une complexité maximale. On y observe de haut en bas la succession suivante (fig. 6):

1 - Sables fins à structure particulaire. Epaisseur 0,35 m. Ils comprennent un horizon sommital légèrement organique de couleur brun jaune pâle (10 YR 6/4) et un horizon basal de couleur jaune (10 YR 7/6). Présence de concrétions ferrugineuses dispersées dans la masse ou concentrées en petites lentilles à la base de la couche. Limite inférieure nette, plane, à pendage faible $\left(6^{\circ}\right)$ vers l'onest.

2 - Sables argileux. Ep. 0,05 m. Structure massive Couleur jaune (10 YR 7/8). Limite inférieure nette plane, a pendage concordant et identique à celui note a la base de la couche 1 .

3 - Argiles sableuses. Ep. 0,35 m. Structure prismatique moyenne. Couleur jaune (10 YR 7/8) et taches rouges très diffuses, devenant de moins en moins nombreuses vers le sommet. Présence de revêtements argileux brun vif (7,5 YR 5/6) sur les faces des agrégats. Limite inférieure du même type que celle des couches supéricures.

4 - Sables argileux, subdivisés en deux horizons:

4a-Bt: Ep. 0,30 m. Structure prismatique grossière.

Couleur panachée: volumes jaunes dominant (10 YR 7)

8) associés à des volumes rouges (2,5 YR 4/6 à 10 R 4/

6 et gris clair $(5$ Y 7/2). Existence de revêtements jaunes (10 YR 7/6) et bruns (7,5 YR 5/4).

4b-Blg: Ep. 0,10 m. Compact à structure prismatiquc

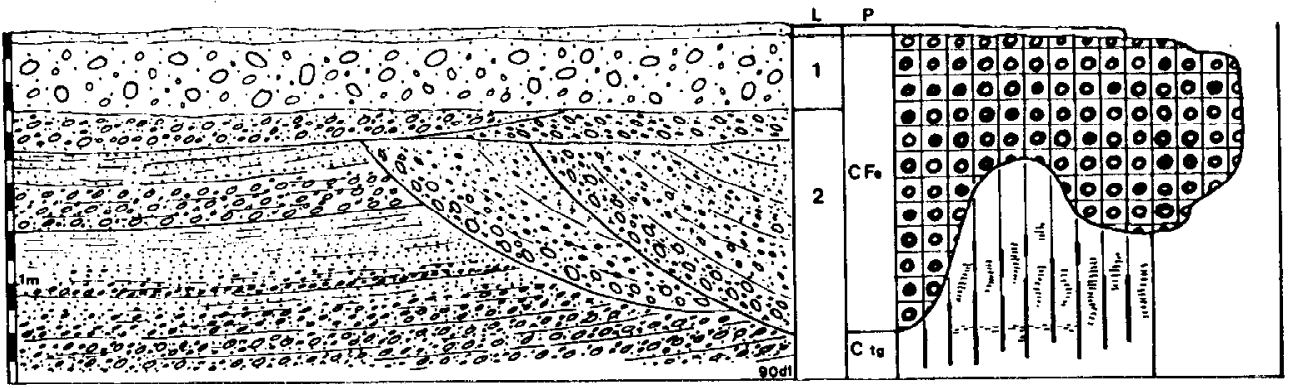

Fìg 4: Litho-pédostratigraphie de la coupe de Salé-Aîn es Sâa.

Fig. 4: Litho-pedostratigraphical section of Sale-Ain es Saa. 


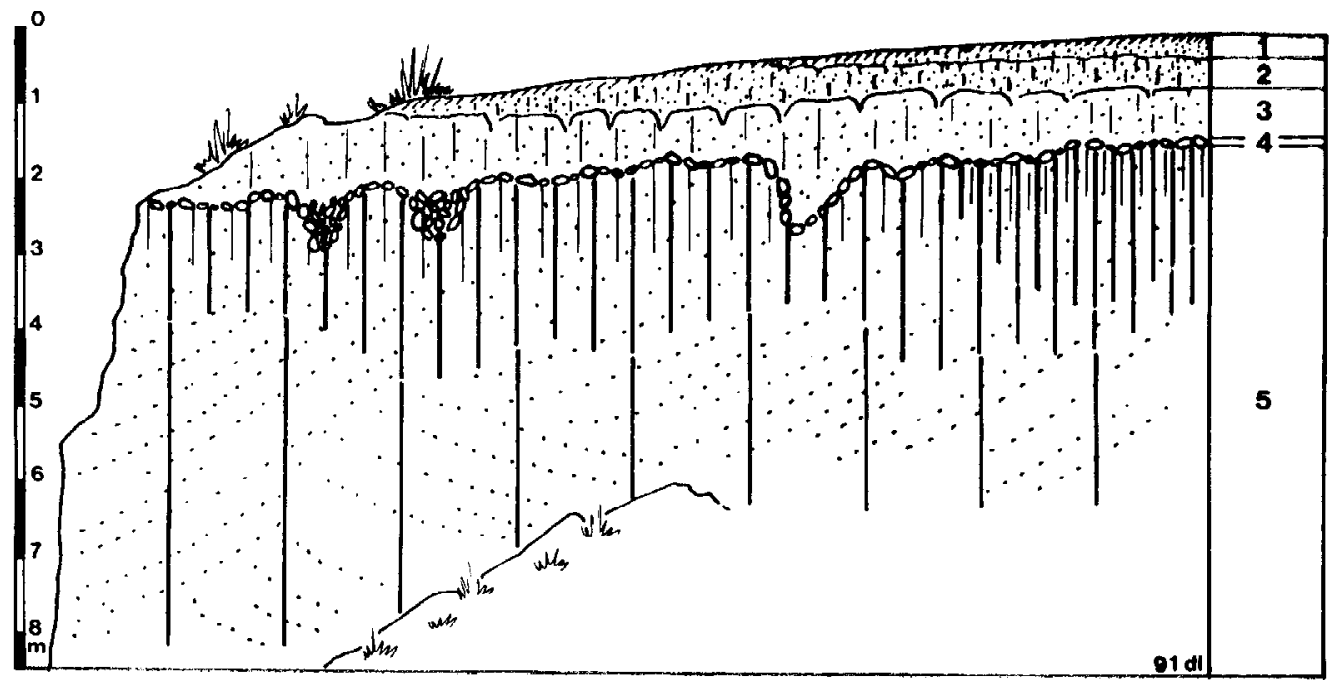

Fig. 5: Lithostratigraphie de la coupe de Salé-ferme de Bayleville.

Fig. Si Litho-pedostratigraphical section of Sale-ferme de Rayteville.

très grossière. Volumes gris-clair $(5 \mathrm{Y} 7 / 2)$ dominant associés à des volumes jaunes. Présence de «slickensides» sur les faces des éléments structuraux. Limite basale brutale, régulière, plane avec un pendage moyen de $8^{\circ}$, soulignée de façon différentielle par l'érosion sur les parois des coupes.

5 - Sables argileux, subdivisés en deux horizons:

5a-Bt: Ep. $0,30 \mathrm{~m}$. Très compact à structure polyédrique grossière. Fond rouge moins vif qu'en $5 \mathrm{~b}$, et taches gris-clair et jaunes. Dans les volumes gris-clair, existence de revêtements jaune pâle. Présence de pisolithes ferrugineux et de graviers dispersés dans la masse.

5b-Btg: Ep. 0,30 m. Structure prismatique bien développec. Couleur panachee, rouge $(10 \mathrm{R} 3 / 6)$, gris-clair (5 Y 7/3) ct jaune (7,5 YR 7/8). Dans les volumes grisclair développement de revêtements jaune pâle ( 5 YR 7/4). Limite inférieure brutale, régulière, plane avec un pendage de l'ordre de $7^{\circ}$, souligné par de petits graviers ou par un liseré gris-clair. Ce plan tronque également le substratum gréseux.

6 - Sables argileux. Epaisseur variable, jusqu'à 1,30 m. Horizon fortement induré à structure massive à prismatique grossière. Couleur rouge vif (10 R 4/8) avec apparition locale de taches gris-clair et jaune. Présence de revêtements rouges sur les faces structurales.

7 - Grès moghrébiens à litage entrecroisé, altérés.

La coupe de Tardiguet-er-Rahla est localisée à environ $2 \mathrm{~km}$ à l'est de la route Tiflet-Aïn Zohra. Très souvent citée dans la littérature, elle a fait l'objet d'études successives (Biberson et al., 1958-59; Biberson, 1961; Saaïdi, 1978; El Hajraoui, 1985; Aberkan, 1989). Des galets à enlevements y ont été recueillis (Biberson et al., op. cit.). Considérant ces objets comme étant d'origine anthropique, Biberson (1961) s'en sert pour définir le stade 1 de son Pré-Acheuléen. Par voie de conséquence, les dépôts auxquels ils sont associés sont attribués au Moulouyen.

La série stratigraphique comporte de haut en bas:

1 - Sables contenant des pisolithes ferrugineux dispersés dans la masse. Ep. moyenne 0,50 m. Structure particulaire. Couleur brun pâle (10 YR 6/4) à jaune pâle (10 YR 7/6). Limite inférieure brutale, rectiligne, faiblement inclinée.

2 - Sables plus ou moins argileux de couleur jaune à la base (10 YR 6/4), visibles sur une douzaine de mètres, fossilifères (nombreux fossiles marins: Pectens, Balanes, dents de requin...) et entrecoupés par plusieurs niveaux de galets subhorizontaux (c'est de l'un d'eux que proviennent les objets attribués au Pré-Acheuléen par Biberson, 1961). Volumes supérieurs altérés et pédogenisés sur une épaisseur plurimétrique ( $8 \mathrm{~m}$ environ) comprenant au sommet un horizon jaune (10 YR 7/8) epais de $0,50 \mathrm{~m}$ et, à la base, un horizon rouge (10 R 4 ) 8 ), conservant quelques fossiles plus ou moins bien conservés (Aberkan, 1989).

\section{5 - INTERPRETATION - DISCUSSION}

5.1 - RELATIONS PEDO-STRATIGRAPHIQUES ENTRE LA FORMATION DE LA MAMORA ET LE SUBSTRATUM «MOGHREBIEN

Dans toutes les coupes étudiées, le contact entre les dépôts rubéfiés considérés comme appartenant à la «Formation de la Mamora» et les dépôts «moghrébiens» francs, est de nature pédologique. Il est généralement matérialisé par un front de décarbonation irrégulier comportant des poches plus ou moins profondes séparées par des chicots carbonatés encore cohérents.

D'autre part, rien ne permet de différencier sédimentologiquement deux unités stratigraphiques. Les structures sédimentaires conservées dans les formations rouges et/ou ferruginisées (Salé - Aïn Saa) sont les mêmes que celles du substratum *moghrébien». D'ailleurs, certains lits caillouteux recoupent le front de décarbonatation et peuvent être suivis de la roche cohérente aux altérites. Les cortèges minéralogiques et pétrographiques sont également très voisins.

Les seules différences constatées sont à mettre sur le compte de phénomènes pédogéniques s'exerçant in situ sur les formations «moghrébiennes»: décarbonatation, 
altération, rubéfaction, constitution d'un épais horizon argiliquc, ferruginisation. Les études micromorphologiques permettent de préciser que l'horizon argilique est complexe et polyphase, et que la rubefaction est liée aux argiles illuviales. De plus, au droit des poches de dissolution, les lits caillouteux hérités de la roche mère sont déformés et ployés en ondulations de largeur généralement métrique.

Par ailleurs, il a déjà été montré (Texier et al., 1984) que le conglomérat servant de stratotype au Salétien correspondait en grande partie (c. 2 de Sale - Ain Saa) à la ferruginisation de la partie supérieure du «Moghrebien» à faciès deltaïque, préalablement décarbonaté et pédogénisé. Seule la partie tout à fait supérieure à structure diamicton (c. 1) peut être considérée comme une colluvion postérieure. La situation morphologique très spécifique de ce site (dépression large et peu profonde, situé en bordure de plateau et ouverte vers la vallée du Bou Regreg) explique très vraisemblablemen ce phénomène de cuirassement unique dans la région.

Dans un certain nombre de coupes (c. 2 de Tardigueter-Rahla et de Salé - Aïn Saa, c. 5 de Sale - Baylevilte) ces transformations sur place de dépôts "moghrébiens" constituent l'essentiel des formations mouluuyennes ou salétiennes des auteurs. Eu égard à l'important volume de dépôts affecté par la pédogénèse (plusicurs mètres ct même parfois plus de $10 \mathrm{~m}$ ), il est probablc que ces phénomènes représentent une importante tranche de temps.

D'autre part, la validité des témoignages «archéologiqucs», qui ont servi de «fossile directeur» pour separer chronologiquement ces formations du substratum, est remise en cause (Raynal et Texier, 1989): les objets inclus dans la couche 2 (substratum "moghrebien" altéré) de Tardiguet (Biberson, 1961) sont vraisemblablement le résultat d'actions naturelles (enlèvement provoqués par l'entrechoquement des galets dans un milieu littoral de haute énergie); ceux de Salé, récoltés hors contexte stratigraphique, proviennent sans doute des colluvions ferruginisées supérieures: ils appartiennent à des phases du Paléolithique nettement plus récentes que celles envisagées par Choubert et Roche (1956) ou Biberson (1971).

\section{2 - LE CARACTERE POLYGENIQUE DE LA PARTIE SUPERIEURE DE LA FORMATION DE LA MAMORA}

Les formations témoignant clairement de phénomènes sédimentaires post- $\gg$ moghrébiens» n'atteignent au mieux que 2 à $3 \mathrm{~m}$ d'épaisseur à la partie superieure de la «Formation de la Mamora". Les données obtenues tant sur le terrain qu'en lames minces montrent le caractère complexe et polyphasé de cette couverture qui a enregistré des phases de stabilite et d'instabilité morphologique ainsi que des événements de nature ubiquiste liés au fonctionnement du karst et à l'hydromorphie (Lefevre et al., 1991).

\section{* Les phases d'instabilité morphologique}

Elles se sont traduites par des phénomènes d'érosion et de sédimentation.

Les phénomènes d'érosion ont occasionné, à l'échelle de l'affleurement, des discontinuités intraformationnelles de plusieurs types:

- surfaces planes obliques (contact entre les différentes couches de Daya el Hamra, limite inferieure de la couche superficielle sableuse de Tardiguet-er-Rahla et de Chaperon-Rouge I et Chaperon-Rouge II),

- lits de galets (c. 4 de Salé-Bayleville),

- contacts ravinants pluridécimétriques à métriques généralement matérialisés par des graviers ou des pisolithes remaniés (c. 2 de Salé-Carrefour et de Chaperon-



Fig, 6: Lithostratigraphie de la coupe de Daya el Hamra

Fig. 6: Litho-pedostratigraphical section of Daya el Hamra. 
Rouge II, localement c. 4 de Salé-Bayleville).

Ces différentes figures ont été interprétees respectivement comme des surfaces de défation, des cailioutis résiduels, des rigoles et des chenaux (Lefèvre $t$ t al., 1991).

Les strates qui constituent la partic supćricurc de la «Formation de la Mamora» (c. 1 de Chaperon Rouge I et de Tardiguet, c. 1 et 2 de Chaperon Rouge II et de Salé-Carrefour, c. 1 de Salé-Aïn Es Sâa, c. 1 à 4 de SaléBayleville, c. 1 à 6 de Daya el Hamra) résultent très nettement de phénomènes d'accrétion. Ceux-ci mettent en jeu deux principaux types de dynamique sédimentaire: colienne ou colluviale.

Ainsi, la couche la plus superficielle de ChaperonRouge I et II, de Tardiguet et de Daya-el-Hamra représente une formation régionale connue sous le vocable de «sables beiges». Or, il a été montré (Texier et Raynal, 1989) que ces derniers correspondaient à un épandage ćolien de type goze, généralisé à tout le nord-ouest du Maroc. Ils se sont édifiés en contexte aride mais en présence d'une végétation éparse qui a piègé les particules sédimentaires en transit et inhibé la formation de dunes. Le maintien d'une certaine activité biologique lors de leur formation est attesté par les chenaux observés en microscopie. Ces sables ont parfois été repris localement par colluvionnement (c. 1 de Salé-Bayleville et de Salé-Carrefour).

D'autres épisodes sédimentaires éoliens se sont manifestement produits anterieurement comme le montrent indirectement les surfaces de deflation qui délimitent les différentes couches de Daya-el-Hamra. Leur préservation ne peut en effet s'expliquer que si la recharge sédimentaire qui les sépare est elle-même de nature colicnne.

La dynamique colluviale est attestée d'une part par les sédiments associés aux structures ravinantes (cf. supra) et, d'autre part, par l'organisation générale des couches rouges et/ou jaunes les plus superficielles. En effet, celles-ci présentent une épaisseur toujours plus importante en bas de versant et dans les creux. Le ruissellement diffus ou concentré représente le mécanisme sédimentaire le plus évident. Néanmoins, l'intervention de phénomènes du type coulées boueuses ou flots de débris demeure très probable même si ceux-ci n'ont pas été démontrés avec certitude jusqu'à présent.

A l'échelle microscopique, on trouve des témoignages de ces phases de remaniement notamment sous la forme de papules et de traits texturaux grossiers dans les vides et les chenaux.

Phénomènes d'érosion et d'accrétion sont à mettre en relation avec un éclaircissement notable du couvert végétal. Ils suggèrent le développement d'un environnement à tendance aride, surtout lors de la mise en place des «sables beiges» sommitaux.

\section{* Les phases de stabilité morphologique}

Ces dépôts sont affectés par des sols de type lessivé rouges et jaunes. En lames minces, ils se caractérisent par des ferriargilanes soit hyalins et finement microlités, soit plus uu moins poussiéreux. Classiquement, la rubéfaction est mise en relation avec un climat contrasté comportant une saison sèche bien marquee (Fédoroff et Courty, 1987), à l'inverse du jaunissement où les oxydes de fer n'ont pas subi de déshydratation. Ccs pédogénćscs attestent de phases de stabilite morphologique ct sont liées à une couverture végétale bien développéc (Fédoroff et Tursina, 1984).

Le nombre d'épisodes rouges lessivés enregistrés est variable selon les sites étudiés. Cependant, quelle que soit la coupe considérée, le sol lessivé jaune se place systématiquement après la dernière phase rouge lessivée et avant le dépôt des «sables beiges» de couverture.

\section{* Les phénomènes ubiquistes}

Outre celles déjà mentionnées dans la partie inférieure de la *Formation de la Mamora* (cf. 5.1), des deformations de plus ou moins grande ampleur ont également affecté les couches superficielles: soutirages des usables beiges» au Chaperon Rouge I et II, déformations en éventail de la structure prismatique du sol lessivé sous-jacent. Ces événements témoignent manifestement de la poursuite de l'évolution du karst sousjacent jusqu'à des périodes très récentes.

D'autre part, un certain nombre de caractères, comme le développement de volumes gris clair et les ferruginisations, sont liés à l'intervention de phases d'hydromorphie dont il est difficile de dire a priori si elles résultent de conditions stationnelles (colmatage des profils, zones de confinement) ou d'evenements climatiques régionaux. Le cuirassement ferrugineux (ferricrète) d'Aïn es Saâ est, quant à lui, lié à une situation morphologique particulière.

\section{3 - LA REALITE DE CYCLES PEDO-SEDIMEN-} TAIRES

Les phases de stabilité et d'instabilité morphologique dont témoigne cette partie supérieure de la «Formation de la Mamora* posent le probleme de l'existence de cycles pédo-sédimentaires répétitifs.

Or, pour ccrtains sitcs (Daya-el-Hamra, ChaperonRouge II), il apparaît que les phascs sćdimcntaircs et pédologiques ne se développent pas de manière aléatoire mais s'ordonnent de manière cyclique de la façon suivante: sol lessivé - hydromorphie - érosion et colluvionnement ou dépôt eolien (El Hajraoui et al., 1984; El Hajraoui, 1985; Raynal et al., 1985; Lefevre et al., 1991) Le caractère répétitif de cette séquence événementielle laisse supposer une commande climatique: climat tempéré humide avec (sol rouge) ou sans (sol jaune) saison sèche marquee, devenant ensuite plus humide (hydromorphie), puis évoluant vers des conditions plus ou moins arides (érosion et remaniement des dépôts plus anciens par colluvionnement et/ou par deflation).

Cependant, l'enregistrement de ces séquences paraît étroitement lié aux conditions stationnelles et, par conséquent, leur nombre varie d'un site a l'autre.

\section{4 - ELEMENTS DE CHRONOLOGIE}

En ce qui concerne la partie supérieure de la «Formation de la Mamora», nous disposons actuellement d'un certain nombre de reperes chronologiques. Les datations numeriques par TL et OSL obtenues sur les «sables beiges» (Texier et al., 1988; Rhodes, 1990; Smith et al., 1990; Raynal et al., 1992) ainsi que les industries préhistoriques (Atérien supérieur, Ibéromaurusien) qu'ils contiennent permettent de situer leur édification entre 28 et 10 Ka environ (Texier et Raynal, 1989).

D'autre part, la couche 2 du Chaperon-Rouge II a éte datée par OSL de 41,1 +/-6.1 Ka (Rhodes, op. cit.). Le sol jaunc lessivé est donc plus ancien puisqu'il est rcmanic par cctte phase colluviale.

Enfin, les dépôts antćricurs a cette phase jaune ont livré de l'Acheuléen (c. 3 de Daya-cl-Hamra) et des industries paleolithiques a choppers et chopping tools (c. 
3 de Chaperon-Rouge II) qu'il n'est pas possible de qualifier avec précision. Ces assemblages archéologiques peuvent donc se situer à l'interieur d'une période allant du dernier Interglaciaire au début du Pléistocène moyen, vers $700-800 \mathrm{Ka}$ (âge des plus anciens témoignages certains de la présence humaine au Maroc, Raynal et Texier, 1989).

La partie inférieure de la "Formation de la Mamora" représente indubitablement la partie alterée et pédogénisée des formations kmoghrébiennesm. Les eléments paleontologiques associés à ces formations permettent de leur attribuer un âge Pliocène moyen à Pliocène supérieur: la base de la série, connue sous le terme de «dalle moghrébienne*, appartient en effet à la biozone a Globonotalia Crassafomis d’âge Pliocène moyen (Wernli, 1978). D'autre part, les faciès fluvio-deltaïques supérieurs ont fourni (gisements de l'oued Fouarat et de l'oued Akrech-Argoub el Hafid) une faune de type Villafranchien inférieur à Mastodonte, Hipparion et Eléphant (Arambourg et Choubert, 1965) et peuvent donc être considérés comme d'âge Pliocène supérieur.

\section{6 - CONCLUSIONS}

Nous n'avons retrouvé aucun témoignage de l'épandage fluviatile des auteurs, censé représenter le Moulouyen regional. En fait, la plus grande partie de la «Formation de la Mamora» correspond à la partie supérieure altérée et pédogénisée des formations «moghrébiennes». De même, le stratotype du Salétien (coupe de Sale-Ain Saa) correspond uniquement à une ferruginisation locale de ces altérites, développée dans un faciès conglomératique et liée à des conditions sta- tionnelles.

La définition des termes chronostratigraphiques «Moulouyen* et *Salétien» repose donc sur une interpretation erronee des facies (confusion entre faciès d'altération et formation sédimentaire). N'exprimant pas une realité stratigraphique, ils doivent etre abandonnes.

En revanche, la partie supérieure de la «Formation de la Mamoran peut être distingué des altérites de la partic basale. Elle résulte en effet de remaniements colluviaux et Eoliens et a enregistré toute une série d'événements morpho-pédo-sédimentaires.

Deux unités stratigraphiques peuvent y être distinguées (Tab. 1). Nous proposons de désigner:

- sous le terme de Formation des sables beiges de couverture, les sables éoliens de couleur claire, datés de 28 à $10 \mathrm{Ka}$.

- sous celui de Formation des sables et argiles rouges panachés de la Mamora, les formations résultant de mécanismes pédo-sédimentaires complexes et dont lâge peut s'étaler du Pléistocène supérieur (à partir du début du dernier cycle climatique) au début du Pléistocène moyen,

Le site de Chaperon Rouge (I et II) peut être retenu comme stratotype de la Formation des sables beiges de couverture. La complexite de la Formation des sables et argiles rouges panachés de la Mamora et la variabilité des enregistrements nous conduisent à choisir pour cette formation un double stratotype représenté par les sites de Chaperon Rouge et Daya el Hamra.

Dans ce même esprit, nous proposons de remplacer les termes d'Argoubien (Biberson, 1971) et de Fouaratien (Stearns, 1978) qui désignaient les formations détri-

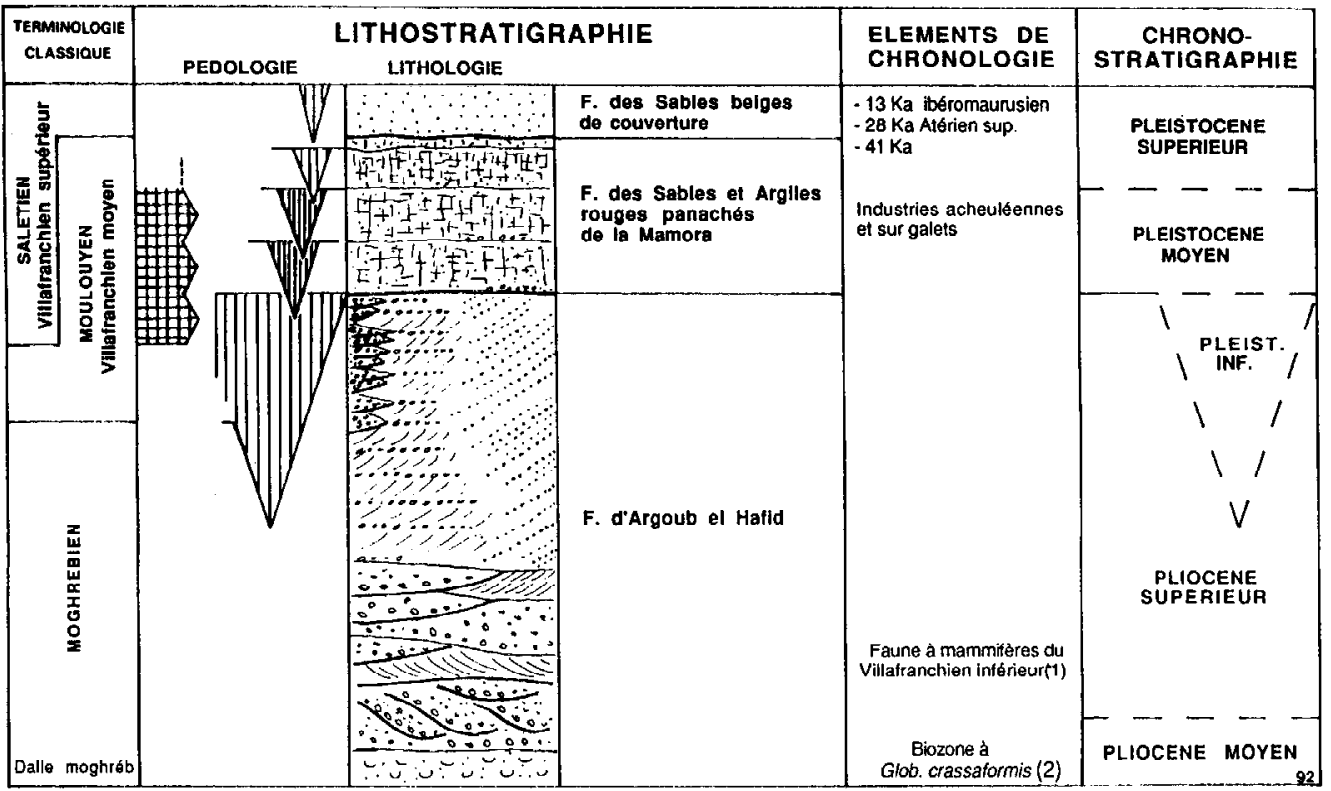

Tab. 1: Lithostratigraphie et chronologie des formations plio-pléistocènes de la région de la Mamora et de Rabat-Salé. (1) d’après les travaux d'Arambourg et Choubert, (2) d'après les travaux de Wernli. Remarques ce tableau ne comprend pas les formations marines et littorales pléistocènes de la trange littorale.

Tab. 1: Lithostratigraphy and chronology of Plio-Pleistocene Formation of the Mamora and Rabat-Sale region. (1) from Arambourg and Chouberh (2) from Wernil. Marine and litoral pleistocene formations of littoral area are not included in this table. 
tiques du substratum reposant sur la «dalle moghrébienne», par celui de Formation d'Argoub el Hafid Nous admettons son stratotype a la localité d'Argoub el Hafid, dans la vallée de l'oued Akrech et son âge Pliocène supérieur (Stearns, 1978).

Le Pléistocène inférieur correspond à une lacune sédimentaire. La puissante altération antérieure à la Formation des sables et argiles rouges panaches de la Mamora et affectant la Formation d'Argoub el Hafid et dont l'âge est compris entre le Pléistocène moyen et le Pliocène supérieur, peut, en partie, lui être rapportée.

\section{REMERCIEMENTS}

Les travaux de terrain ont été réalisés dans le cadre de la Mission préhistorique et paléntolugique française au Maroc et financés par le Ministère des Affaires Etrangères. Ils ont été facilités par l'aide apportée sur place par les Autorités marocaines de tutcllc (Ministère de la Culture, INSAP) et par le Service culturel de l'Ambassade de France. Parmi le personnel de ce servicc, nous tenons à remercier plus particulièrement madame D. Penot pour son efficacite et son appui amical. Nous sommes également reconnaissant à monsieur J. Sommé de sa lecture critique du manuscrit et de ses suggestions avisées qui ont contribué à l'amélioration du texte présenté ici.

\section{BIBLIOGRAPHIE} ABERKAN, M., 1989 - Enude des formations quaternaires des marges
du bussin du Rharb (Maroc nord octidental). Thise, Universit $E$ de Bordeaux $1,290 \mathrm{p}$.

ANDRE, A et BEAUDET, G, 1967 - Observations nouvelles sur lcs depbts quatemaires des environs de Rabat. Rev. Géogr. Maroc, 11, $77-98$

ARAMBOURG, $C$. et CHOUBERT, $G_{n} 1965$ - Les faunes de Mammifères de l'étage Moghrébien du Maroc occidental. Notes Serv. géol. Maroc, 25, 185, 29-33.

BEAUDET, G, MAURER, G, RUELLAN, A, 1967 - Le Quaternaire marocain. Observations et hypothèses nouvelles. Rev. Géogr. Phys. Gévl. Dyn., DX, 4, 269-310.

BEAUDET, G, 1969 . Le Plateau central marocain et ses bordisres. Euude géomorphulogique. Inf ramar, Rabat, $478 \mathrm{p}$.

BEAUDET, G, 1971 - Le Quaternaire marocain: état des études. Rev. géogr. Maroc, 20, 3-55.

BIBERSON, $\mathrm{P}_{4} 1961$ - Le cadre paleogéographique de la préhistoire ou Maroc atlantique. Publ. Serv. Antiq. Muroc, 16, Ralbat, 235 p. BIBERSON, $P, 1971$ - Essai de redéfinition descycles climatiques du Quaternaire continental du Maroc, Bull. Ass. Fr. Er. Quat., 1, 3-13. qumologie méditerraneenne. Bull. Soc visions sur la base de
Géol. Fr., 17, 380-393.

BULLOCK, P, FEDURUF, N, JONGERIUS, A, STOOPS, G. Publ., Wolverhampton, $152 \mathrm{p}$.

CHOUDERT, G, 1957-65a - Essai de corrélation des formations Maroc, 25, 185, 35-45.

CHOUBERT, G, 1957-65b - L'étage Moghrébien dans le Maroc occidental. Notes Serv. géol. Maroc, 25, 185, 47-55.

CHOUBERT, G., 1965 - Le Quaternaire au Mamc. Notes Serv. géol. Maroc, 25, 185, 9-27.

CHOUBERT, G, JOLY, F, GIGOUT, M., MARCAIS, J, MARGAT J. et RAYNAL, R, 1956 - Essai de classification du Quaternaire continental du Maroc. C. R. Acad. Sci. Paris, 243, 504-506.
CHOUBERT, G., et ROCHE, J., 1956 - Notes sur les industries anciennes du plateau de Salé. Bull. Archéol. Maroc, 1, 9-37.

CIRAC, $P_{,} 1985$ - Le Bassin sud-rifain occidental. Evolution de la dynamique sédimentaire et de la paléogégraphie au cours d'une phase de comblement. Thèse Sriences, Univ. Rordeaux 1, 2 t., 285

EL HAJRAOUI, MA., 1985 - Les industries préhistoriques de la région de la Mamora dans leur contexte géologique et palépédogique.
Thése 3ème cycle, Université de Bordeaux 1, 185 p.

EL HAJRAOUI, MA, FEDOROFF, N, RAYNAI, J.P. et TEXIER, J.P., 1984 - Nouvelles donnces sur les paléoenvironnements de la Mamora (Maroc): Étude paléptologique de la sequence de Fr. Ed., 210.

FARA, H., 1963 . Enude pédologique des sols de la Mamora. Inédit, 110 p., 1 carte, I.N.R.A., Rabat.

FEDOROFF, N. et COURTY, M.A., 1987 - Palt́osols. In: Génlogie de la Préhistoire, J.C. Miskovsky Ed., 251-280.

FEDOROFF, N. et TURSINA, T., 1984 - Micromorphologie des sols lessivés de France et des sols dernovopodzoliques d'Union Soviétique. Polycopié INA-PG, 32 p.

GIGOUT, M, 1958 - Sur le Pliocène et le Quatemaire de Rabat et de Sale CR Acad Sci Paris, 247, 17, 1363-1366.

GIGOUT, M., 1960 - Nouvelles recherches sur le Quaternaire marocain et comparaison avec l'Europe. Trav. Lab. géol. fac. sci. Lyon, 6, $158 \mathrm{p}$.

GIGOUT, M. et RAYNAJ, R, 1957 - Corrélation des phénomènes marins et continentaux dans le Qualermaire marocain. C. R Acad. Sci. Paris, 244, 20, 2528-2531.

GIGOUT, M. et RAYNAL, R, 1959 - Retouche à la currélation des phénomenes marins et continentaux dans le Quatemaire marocain. C. $R$. Acad. Sci. Paris, 248, 15, 2223-2225.

HEDBERG, H.D, 1976 - Intemational Stratigraphic Guide, A guide to tratigraphic classification, Lerminology and procedure. Wiley, NewYork, 200 p. Trad. Pr, 1979, Guide strationghique inemational P.

LE COZ, J, 1964 - Le Rharb. Fellahs et coluns. Etude de geographie régionale, 1, 482 p., Rabat.

LEFEVRE, D, TEXIER, J.P. et RAYNAI, J.P, 1991 - Organisation et dynamique d'un complexe pédo-sédimentaire: la Formation de ture pédologique et son importance pour la compréhension de la morphogénese, Caen, résumé.

LEPOUTRE, 1966-68 - La Mamora. Congrès de Pédologie méditerra néenne, excursion Maroc, Al Awamia, $279-295$.

LHENAFF, R, Ed, 1983 - Le Villafranchien mediterranéen. Stratigraphie, environnement bioclimatique, morphugenèse et néotecgraphie, environnement bioclimatique, morphugenese
tonique, Colloq. Lille, 1982, Bull. Ass. Fr. Et. Quat., 2 -3.

RAT, P., 1980 - Méthodologie stratigraphique et Quaternaire. In "Problemes de stratigraphie quaternaire en France et dans les pays limitrophes, Dijon, Suppl. Bull. Ass. Fr. Et. Quat., 1, 4-14.

RAYNAI, J.P, TEXIER, J.P. of DEBENATH, A, 1982 - Les limons rouges de la Meseta côtière maracaine: limites et chronologies, Ed., 535 .

RAYNAI, J.P,FEDOROFF, N, TEXIER, J.P.et EL HAJRAOUI, A 1985 - Genése des horizons argiliques rouges et jaunes au Maroc atlantique (Mamora). Réun. Intern. micromorphologie des sols, Paris, résumés, 169

RAYNAL, J.P. et TEXIER, J.P, 1989 - Découverte d'Acheuléen ancien dans la carrière Thomas 1 a Casablanca et problème de Paris, 308, II, 1743-1749.

RAYNAL, J.P, TEXIER, J.P, LEFEVRE, D. et RHODES, E, 1992 . Les Sables Beiges de Couverture et l'Atérien en Mamora, nouveaux eléments de chronologie numerique. Colloq. Thomme de

RHODES, EJ, 1990 - Optical Dating of Quartz from Sediments. These de Doctorat, Univ. d'Oxford, 153 p.

SAAIDI, E.K, 1974 - Enude geomorphologique de la région de Tiflet et des conditions morphostricturales et sedimentologiques de la gent's de la formation de la Mamora. D.E.S. 3tme cycle, Univ. Moham-
med V, Rabat. 
SMITH, B.W, RHODES, EJ, STOCKES, S, SPOONER, N.A. and ATTKEN, M J 1990 - Optical Dating of Sediments: In, N.A. and Results from Oxford. Archaeometry, 32, 19-31.

STEARNS, C.E. 1978 - Pliccene-Pleistocene emergence of Maroccan Meseta. Geol. Soc. Amer. Bull., 89, 1630-1644.

TEXIER, J.P., 1985-86 - Le site atérien du Chaperon-Rouge I (Maroc) et son contexte géologique. Bull. d'Archéol. Marocaine. VI, 27-74.

TEXIER, J.P, DEBENATH, A, et RAYNAL, J.P. 1982 - Une stratigraphie complexe du Quaternaire continental marocain: le Chape-
ron Rouge if à Rabut. Seme Rén. an. Sci. Terre, Paris, Soc. Géol. Fr. Ed., 399 .

TEXIER, J.P., RAYNAI, J.P, LEFEVRE, D. et FEDOROFF, N. 1984 - De la validite des deux plus anciens etages continentaux du
Scl. Terre, Bordeaux, Soc. Geol. Fr. Ed., 523.

TEXIER, J.P, RAYNAL, J.P. et LEFEVRE, D, 1985 - Nouvelles propositions pour un cadre chronologique raisonne du Quater-

TEXIER, J.P, HUXTABLE, J, RHODES, E.J, MLALIER, D., et OUSMOI, M, 1988 - Nouvelles donnés sur la situation chronologique de l'Atérien au Maroc et leurs implications. C. R. Acad.

TEXIER, J.P. et RAYNA , J.P. 1989 - Les ksables beiges* du NordOuest du Maroc: nouvelles interprétations dynamiques, chronolo-
giques et paléclimatiques. C. $R$ Acad. Sci. Paris, 309, II, 1577
is82. 1582.

WERNLI, R, 1978 - La base du Moghrebien est d'age Pliocène moyen (zone da. Grassaformis) dans la Mamora (Maroc). Arch 